\title{
SINCRONIZAÇÃO DE SEMÁFOROS: MODELO MATEMÁTICO PARA UMA APLICAÇÃO AO TRÁFEGO DE CURITIBA
}

\author{
S. Joner' e N. M. P. Volpi ${ }^{2}$ \\ ${ }^{1}$ Campus Geraldo Werninghaus - Instituto Federal de Santa Catarina \\ ${ }^{2}$ Depto de Matemática - Universidade Federal do Paraná \\ sander.jonerl@ifsc.edu.br - mnpv@mat.ufpr.br
}

Artigo submetido em fevereiro/2013 e aceito em abril/2013

\section{RESUMO}

Em função do crescimento das cidades e consequentemente do número de semáforos se faz necessário uma abordagem científica na sincronização dos mesmos. Entende-se por sincronização, a defasagem entre os centros dos vermelhos dos semáforos de uma região, de modo que os estabelecimentos dos tempos de verde e vermelho sejam tais que, um motorista trafegando numa via encontre sinais verdes ao longo dela. A porção de um ciclo de um semáforo para o qual isto é possível denomina-se largura de banda. O objetivo deste trabalho é aplicar um modelo matemático computacional para sincronizar semáforos, objetivando maximizar largura de banda ao longo das artérias mais importante e particularizada para a situação do tráfego em Curitiba. Tal abordagem acarretaria em economia, qualidade de vida e segurança no trânsito. O modelo aplicado baseia-se nos trabalhos de (LITTLE, 1966) e (GARTNER, 1981), de programação linear inteira mista e envolve restrições de ciclo, restrições de velocidade, de mudança de velocidade entre trechos, com tempos de espera para virar à esquerda e tempo de espera do pelotão semafórico.

PALAVRAS-CHAVE: largura de banda, sincronização e programação linear inteira mista.

\section{SYNCHRONIZING TRAFFIC LIGHTS: MATHEMATICAL MODEL FOR AN APPLICATION TO TRAFFIC CURITIBA}

\begin{abstract}
Due to the city growth and consequently to the increasing number of the traffic signals, a scientific approach is necessary to establish the synchronization among them. Synchronization means the relative phase of signals of a region such that, the fraction of the green and red time of a cycle are such that, a driver has uninterrupted flow traffic throughout a main arterial. The portion of the cycle for which this is possible is called bandwidth. The main goal of this work is to develop a computational mathematical model to synchronize traffic signals so that the bandwidth can be
\end{abstract}

as big as possible throughout the most important avenues. Such model is being developed specifically to Curitiba traffic network. Such approach implies in saving money and time, improving the quality of life and making the streets safer. This model is based on the works of (LITTLE,1966) and (GARTNER,1981), about mixed integer linear programming and contains restrictions of cycles, speed, changing speed among signals, protecting left-turn movements and considering the time to wait at the signals platoons. Some tests are being done at sub areas in Curitiba.

KEY-WORDS: bandwidth, synchronization and mixed integer linear programming. 


\section{SINCRONIZAÇÃO DE SEMÁFOROS: MODELO MATEMÁTICO PARA UMA APLICAÇÃO AO TRÁFEGO DE CURITIBA}

\section{INTRODUÇÃO}

\subsection{Identificação e Caracterização do Problema}

Em decorrência da urbanização acelerada e desorganizada, um dos problemas que mais afeta as grandes cidades é de como fazer o trânsito de pessoas, veículos e mercadorias fluir de maneira harmoniosa.

O transporte rodoviário domina o mercado nacional, seja no espaço rural ou no urbano. Estima-se que no Brasil $96 \%$ das viagens de pessoas e $64 \%$ das cargas transportadas ocorram em rodovias. Logo, o transporte rodoviário, no total, consome muito mais energia que qualquer outro tipo de transporte. O número de veículos automotivos no país tem crescido rapidamente nas últimas décadas, passando de 430.000 em 1950 para 3,1 milhões em 1970 e chegando a 25 milhões em 1995. Atualmente, a frota está em cerca de 29 milhões de veículos, conforme (PNT: Política Nacional de Trânsito) e (GARCIA ,1995).

Uma das formas de promover a harmonia no trânsito, melhorando a mobilidade de pessoas e veículos, é a sincronização dos semáforos, onde faz-se uma abordagem científica dos tempos de verde e vermelho dos mesmos. A suavização do tráfego nas cidades melhoraria a segurança do trânsito e a qualidade de vida dos habitantes. Melhorar a qualidade de vida em relação ao trânsito significa promover melhores condições de acessibilidade de pessoas e mercadorias, evitar problemas ambientais e destruição do patrimônio histórico devido à abertura de novas vias, diminuir a irritabilidade do motorista em relação ao trânsito caótico, evitando até muitos acidentes, enfim, repelir qualquer mal que possa atrapalhar os cidadãos no presente ou no futuro.

Já no quesito segurança, a principal meta seria evitar acidentes, embora não se resuma a essa finalidade. Os pedestres, principalmente idosos e deficientes, devem ter as mesmas prioridades que os veículos de se locomoverem, pois representam uma grande parcela da população. E os veículos devem ter uma movimentação contínua sobre as vias, para chegarem aos seus destinos no menor tempo possível, sem exceder a velocidade máxima imposta por lei. Com os veículos em constante movimento evitam-se assaltos, bloqueios em cruzamentos e congestionamentos, melhorando a fluidez do tráfego, o conforto e a segurança dos usuários do sistema viário.

Economicamente, o país também perde com o atual estado do trânsito. Estima-se que os gastos resultantes do congestionamento nas capitais (quando a capacidade das vias está esgotada) atingem a cifra absurda de R\$ 450 milhões ao ano (BRASIL, 2011). Caso fossem incluídas no cálculo as outras cidades médias e grandes, o valor chegaria à casa dos bilhões de reais anuais, sem contar as perdas provocadas pelos acidentes.

Vários países já desenvolveram estudos sobre a sincronização de semáforos, comprovando sua importância e eficiência. Contudo, esses trabalhos envolvem aplicações aos casos de seus respectivos países de origem. Poucos trabalhos brasileiros são apresentados na bibliografia da área, por isso é importante desenvolver estudos e modelos considerando as 
particularidades do tráfego regional brasileiro. Essa é justamente a proposta apresentada: a partir dos modelos já testados em outros países, apresentar um modelo adequado à realidade brasileira.

Em contatos feitos com a DIRETRAN em Curitiba, foi confirmado que seria muito útil discutir e apresentar sugestões sobre uma maneira de sincronizar semáforos, e que tal medida acarretaria em economia, melhoria na mobilidade urbana e qualidade de vida e segurança no trânsito.

\subsection{Objetivo}

Desenvolver uma proposta de modelo matemático computacional para sincronizar os semáforos de uma malha viária. O tempo que o motorista tem para atravessar uma via (artéria) encontrando verde em todos os sinais deve ser o maior possível, dentro dos limites de velocidade e adequado ao fluxo de pedestres. A ideia é que os carros que trafegarem com velocidade dentro de um certo intervalo preestabelecido não fiquem parados nos semáforos, e que no tempo de vermelho, os pedestres (lembrando dos idosos e deficientes) possam atravessar a faixa com segurança. Isto gera segurança no trânsito e bem-estar para todos.

\section{REVISÃO DA LITERATURA}

$\mathrm{Na}$ literatura especializada existem vários enfoques para resolver tal situação. Alguns trabalhos são considerados clássicos como o de Morgan e Little (1964), e Little (1966). A partir destes, alguns programas foram desenvolvidos como o TRANSYT e MAXBAND, citados nos trabalhos de Cohen (1986) e Little et. al. (1981). Outros enfoques também podem ser encontrados nos trabalhos de Cohen e Pillai (1998), Gartner (1972), Gartner et. al. (1975) e Porto Jr. e Ferreira (1993). A maior parte dos trabalhos citados apresentam estudos de casos de seus países de origem.

\section{DESENVOLVIMENTO DA PROPOSTA}

\subsection{Metodologia}

A metodologia usada será avaliar as possibilidades de solução em função dos artigos revistos, e então será proposto um trabalho adequado à realidade brasileira com possibilidades de implantação do sistema. As etapas consideradas serão:

- Formular e desenvolver um modelo de programação linear inteira mista e resolvê-lo pelo método de Branch and Bound e heurística, caso necessário.

- Escolher as principais vias de uma região (cidade) e seus respectivos semáforos a serem sincronizados. 
- Testar o modelo formulado, desenvolvendo um programa para a sua execução, aplicável na solução direta do problema.

- Aplicar o modelo à região escolhida, almejando o melhor resultado possível.

Para desenvolver as etapas citadas, exige-se o conhecimento de:

- Informações das artérias principais da cidade;

- Informações do número de semáforos de cada artéria e de sua posição geográfica;

- Limite superior e inferior no período do semáforo;

- Limite superior e inferior nas velocidades permitidas nas artérias;

- Limites permitidos de mudança de velocidade nos trajetos compreendidos entre os semáforos.

Com esses dados e através da execução do modelo proposto, pode-se encontrar:

- Um período comum para todos os semáforos, caso necessário;

- Tempos de verde e vermelho de cada semáforo;

- Velocidade e tempo gasto entre os semáforos;

- Diferença de fases entre os semáforos.

A seguir, será descrito como o problema de sincronização dos semáforos será resolvido. Inicialmente, abordar-se-á a sincronização de uma artéria, e depois da malha viária toda, considerando as interseções das artérias principais.

\section{A sincronização dos semáforos:}

Define-se a sincronização dos semáforos como o conjunto de valores $\theta\left(j_{1}, j_{2}\right)$, medido pelo tempo entre o centro do vermelho de $S_{j 1}$ até o próximo centro de vermelho de $S_{j 2}$, que é a fase relativa entre os semáforos $S_{j 1}$ e $S_{j 2}$.

Desta forma, o objetivo é encontrar a fase relativa entre os semáforos, de modo que o fluxo de pedestres e veículos ocorra mais suavemente.

\section{O problema da artéria:}

O termo artéria será usado para referir-se a qualquer rua onde a progressão é desejada, isto é, da qual é definida uma largura de banda. A porção de um ciclo do semáforo onde um carro, saindo da extremidade de uma artéria principal e se deslocando com uma certa velocidade, passa de semáforo em semáforo sem parar em sinal vermelho, é definida como a largura de banda (bandwidth).

Será considerada uma rua dupla tendo $n$ semáforos. Os sentidos da rua são identificados como inbound e outbound. Os sinais serão denotados por $\mathrm{S}_{1}, \mathrm{~S}_{2}, \ldots, \mathrm{S}_{\mathrm{n}}$ no sentido outbound.

O conjunto de possíveis trajetórias desimpedidas em um dado sentido formam uma banda verde (green band), da qual a largura horizontal é a largura de banda naquele sentido. 
O programa matemático proposto maximizará a soma das larguras de banda nos dois sentidos da artéria, sujeito a restrições de limites de velocidade, limites de taxas de variação de velocidade nos trechos compreendidos entre semáforos e restrições inteiras de avaliações dos ciclos.

O problema da rede viária:

O problema da rede viária consiste em problemas arteriais para ruas individuais mais as restrições de ciclos que conectam as ruas que se interceptam.

O objetivo, neste caso, será maximizar as larguras de banda de todas as artérias da rede, utilizando de ponderação para garantir o grau de importância entre as artérias.

Um conjunto de restrições, chamado de restrições de ciclos, é introduzido sempre que várias artérias se interceptam para formar um ciclo fechado (loop).

A terminologia da Teoria dos Grafos pode ser usada para descrever as redes, como em Wilson e Watkins (1990). Semáforos são denominados de nós. Comprimento das ruas entre sinais adjacentes são arcos ou extremidades. Cada arco tem um sentido e é indicado por uma flecha. A seqüência de arcos formará um ciclo.

Um semáforo na interseção de duas artérias pode ser crítico para uma artéria mas não para a outra. Por crítico entende-se aquele semáforo cuja largura de banda não pode ser maior porque o tempo de verde do mesmo não permite. Então, a alteração do tempo de verde de uma rua em um cruzamento pode aumentar a largura de banda em uma artéria sem reduzir a da outra. Assim, o tempo de vermelho nas interseções arteriais pode, às vezes, ser uma variável de decisão útil. A fração de tempo de vermelho em cada rua pode ser limitada em função do fluxo. O tempo do vermelho também pode ser limitado para permitir uma travessia segura dos pedestres e, ao mesmo tempo, solucionar o tempo de espera do motorista. Todos esses fatos serão descritos nas restrições denominadas tempo de vermelho (red-time).

Então, para resolver um problema da rede viária, seguem-se os seguintes passos:

- Determinar quais das ruas da rede serão as artérias principais;

- Descrever a função objetivo e as restrições para cada artéria principal;

- Descrever a restrição de período dos semáforos;

- Descrever as restrições inteiras de ciclos entre dois semáforos consecutivos;

- Descrever as restrições de ciclos das artérias que formam um loop;

- Adicionar restrições tempo de vermelho.

\subsection{Modelo matemático}

A seguir, serão descritas as equações matemáticas que geram as variáveis de controle, as quais permitem a sincronização dos semáforos de uma rede.

O modelo matemático apresentado consiste em:

- Descrever equações para as artérias individualmente;

- Descrever a função objetivo, que será a soma ponderada das larguras de banda de todas as artérias da rede, garantindo uma ordem de importância entre as artérias; 
- Descrever as restrições de ciclos;

- Descrever as restrições tempo de vermelho de controle dos tempos de movimento e espera.

Dados do modelo:

$T_{1}, T_{2}: \quad$ valores mínimo e máximo dos períodos permitidos;

$v_{\min }^{i}, v_{\max }^{i}: \quad$ valores mínimo e máximo das velocidades permitidas no sentido outbound;

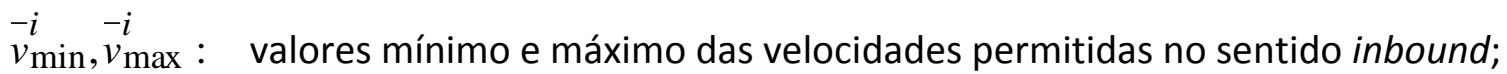

$a_{i}$ : grau de prioridade da artéria $i$ relativo à artéria de referência, artéria 0 , considerada a mais importante da rede;

$d_{i}\left(j_{1}, j_{2}\right)$ : distância entre os semáforos $S_{j 1}$ e $S_{j 2}$ da artéria $i$

$L: \quad$ número de ciclos independentes da malha viária;

$q$ : $\quad$ número de artérias principais da malha viária;

$J_{i}: \quad$ número de semáforos da artéria $i ;$

$S_{i j}: \quad$ semáforo $j$ da artéria $i$

$R_{M I M}$ : $\quad$ valor mínimo do tempo de vermelho permitido nos sentidos outbound e inbound da artéria $i$, do semáforo $j$.

$R M A X_{i j}$ : valor máximo do tempo de vermelho permitido nos sentidos outbound e inbound da artéria $i$, do semáforo $j$.

Variáveis de decisão e controle:

$b_{i}: \quad$ largura de banda da artéria $i$

$z: \quad$ freqüência dos sinaleiros;

$v_{i}: \quad \quad \quad$ velocidade do veículo na artéria $i ;$

$u_{i}=\frac{1}{v_{i}}: \quad$ velocidade recíproca do veículo na artéria $i$

$w_{i j}$ : $\quad$ tempo de folga após a abertura do verde, que o motorista tem para entrar na banda verde da artéria no sinaleiro $j$ da artéria $i$;

$t\left(j_{1}, j_{2}\right)$ : $\quad$ tempo gasto pelo veículo para ir de $S_{j 1}$ até o $S_{j 2}$; 
$m\left(j_{1}, j_{2}\right)$ : tempo que o motorista leva para sair do centro do vermelho do semáforo $S_{j 1}$ e voltar para o mesmo ponto passando pelo semáforo adjacente $S_{j 2}$;

$c\left(j_{1}, \ldots, j_{p}\right):$ tempo que o motorista tem para percorrer um loop fechado partindo do centro do vermelho de $S_{j 1}$ e chegando no mesmo semáforo após passar por $S_{j 2}, S_{j 3}, \ldots, S_{j p}$ . , sendo que os semáforos considerados são só os das interseções das artérias que compõe o loop;

$r_{i j}: \quad \quad \quad$ tempo de vermelho do semáforo $j$ na artéria $i$.

Todos os tempos são medidos em ciclos do período usado nos semáforos.

Na Figura 1 estão representados os dados e as variáveis de decisão.

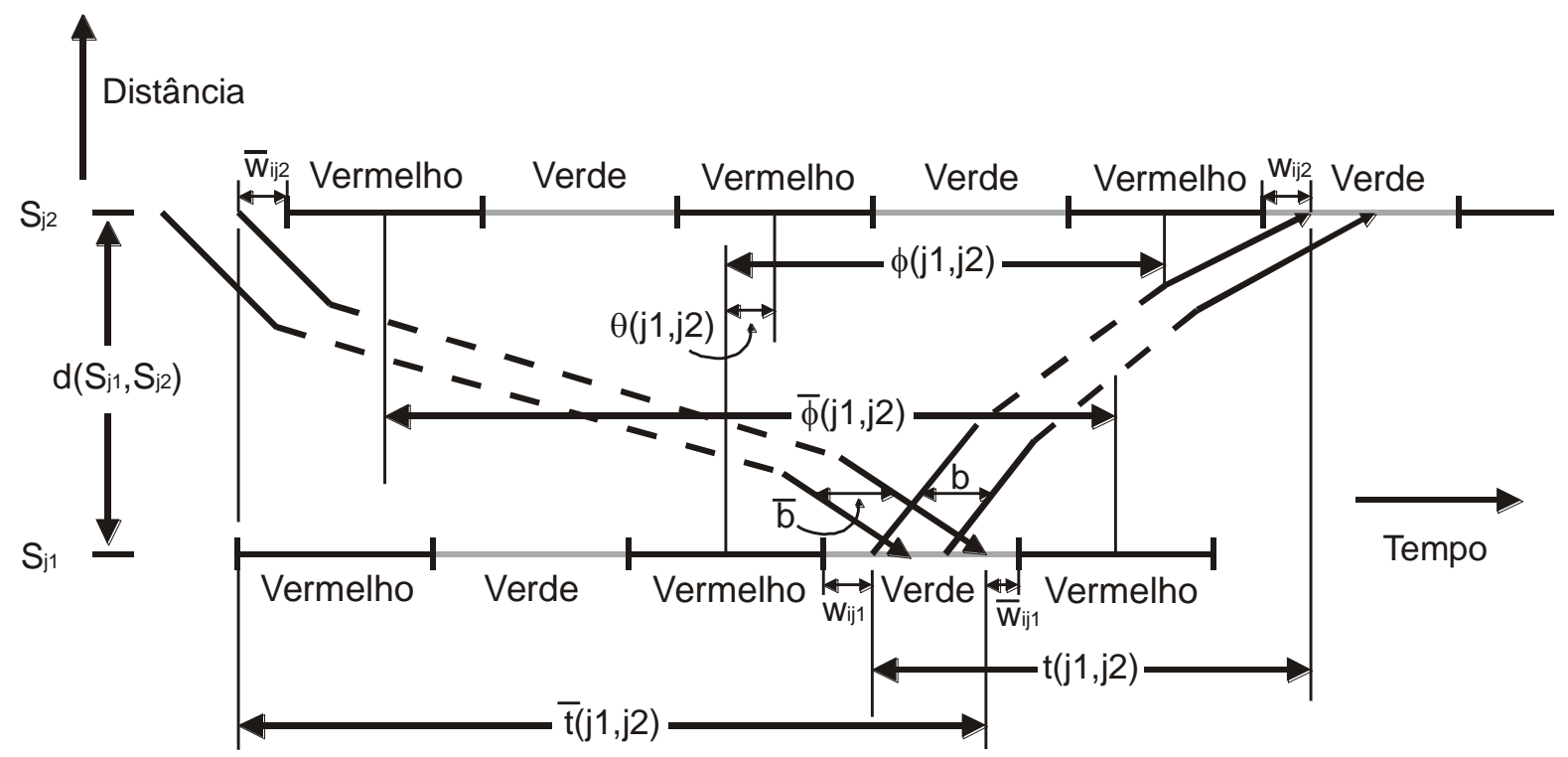

Figura 1: Representação dos dados e variáveis de decisão

\section{Função objetivo do modelo:}

Maximizar as larguras de banda de todas as artérias principais ponderadas ao grau de prioridade entre as artérias consideradas.

$$
\max b_{0}+a_{1} b_{1}+\ldots+a_{q} b_{q}
$$

\section{Restrições do modelo:}

(1) Restrições de proporcionalidade das larguras de banda de cada artéria i, $\quad 1 \leq i \leq q$. 
São consideradas $q$ restrições que limitam os valores de largura de banda à proporções adequadas, evitando grandes diferenças entre as larguras de banda, relativamente a uma artéria principal, artéria 0 , de largura de banda $b_{0}$.

$b_{i} \geq a_{i} b_{0} \quad, i=1, \ldots, q$

(2) Restrições de limite de frequência dos semáforos.

Neste modelo está sendo considerada uma freqüência única para todos os semáforos, limitada a valores preestabelecidos $T_{1}$ e $T_{2}$. De uma forma geral consideram-se grupos de semáforos com mesma freqüência.

$1 / T_{2} \leq z \leq 1 / T_{1}$

(3) Restrições de velocidade nos trechos entre os semáforos de cada artéria $i$.

O Código Nacional de Trânsito (CNT) prevê limites de velocidade nas artérias, (CNT- Código Nacional de Trânsito) e para o bom andamento do fluxo uma velocidade mínima é necessária, assim, limita-se a velocidade do veículo na artéria $i$, através das restrições:

$$
v_{\min }^{i} \leq v_{i} \leq v_{\max }^{i} \quad i=0, \ldots, q
$$

Como o tempo será medido em ciclos e $T=\frac{1}{z}$, tal restrição pode ser reescrita por:

$z\left(\frac{1}{v_{\max }^{i}}\right) \leq u_{i} \leq\left(\frac{1}{v_{\min }^{i}}\right) z, \forall i=0, \ldots, q$ no sentido outbound

onde $u_{i}$ é a velocidade recíproca multiplicada pela freqüência, medida em ciclos/metro, representando quantos ciclos do semáforo $S_{j}$ ocorrem por metro de deslocamento do veículo até o mesmo.

Da mesma forma, no sentido inbound, existem mais outras $q+1$ restrições da forma:

$z\left(\frac{1}{v_{\max }}\right) \leq \bar{u}_{i} \leq \underset{v_{\min }}{\left(\frac{1}{-i}\right)}, \forall i=0, \ldots, q$ 
(4) Restrições de tempo, para cada semáforo $S_{j}$ de cada artéria $i$

$$
w_{i j}+b_{i}+r_{i j} \leq 1, \quad \forall i=0, \ldots, q, \quad \forall j=1, \ldots, J_{i}
$$

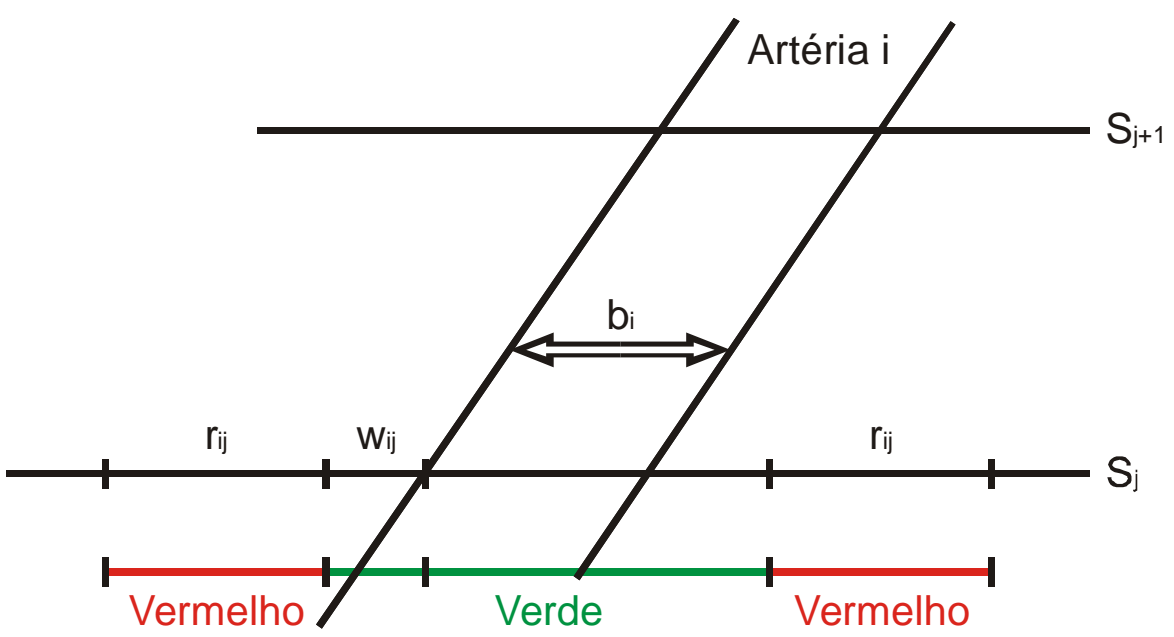

Figura 2: Representação das variáveis nas restrições de tempo

Esta restrição diz que a folga do tempo do verde para entrar na banda, mais o tempo de banda, mais o tempo de vermelho, não deve ultrapassar um ciclo (tempo de verde mais vermelho).

Da mesma forma, no sentido inbound,

$\bar{w}_{i j}+b_{i}+\bar{r}_{i j} \leq 1, \quad \forall i=0, \ldots, q, \quad \forall j=1, \ldots, J_{i}$

(5) Restrições inteiras para cada semáforo $S_{j}$ de cada artéria $i$

$$
\begin{aligned}
& \left(w_{i j_{1}}+\bar{w}_{i j_{1}}\right)-\left(w_{i j_{2}}+\bar{w}_{i j_{2}}\right)+d\left(j_{1}, j_{2}\right)\left(u_{i}+\bar{u}_{i}\right)+r_{i j_{1}}-r_{i j_{2}}=m\left(j_{1}, j_{2}\right) \\
& \forall i=0, \ldots, q, \quad \forall j=1, \ldots, J_{i}
\end{aligned}
$$




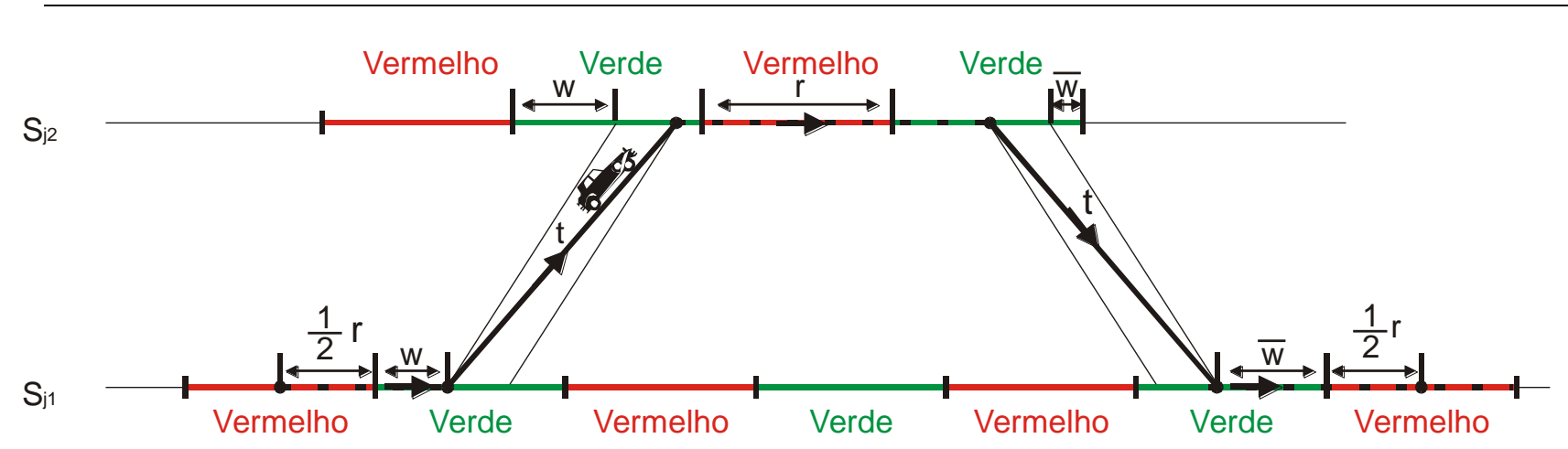

Figura 3: Representação das variáveis nas restrições inteiras.

Esta restrição representa o número de ciclos ou o tempo que um veículo levaria para percorrer o trajeto entre o centro do vermelho do semáforo $S_{j 1}$ e o centro do vermelho do semáforo adjacente $S_{j 2}$ e retornar ao centro do vermelho do semáforo $S_{j 1}$ inicial.

(6) Restrições de ciclos

Supõe-se que o número $L$ de ciclos da malha seja conhecido. O número de restrições de ciclos necessário é menor que o número de ciclos (equivale ao número de áreas distintas envolvidas pelas artérias do loop);

Para cada $l=1, \ldots, L$, considera-se uma restrição da forma:

$c_{l}\left(j_{1}, \ldots, j_{p}\right)=\phi\left(j_{1}, j_{2}\right)+\ldots+\phi\left(j_{p}, j_{1}\right)+1 / 2 p$

Onde:

$S_{j_{k}}$ são os semáforos de interseção das artérias do loop $I, \phi\left(j_{k}, j_{m}\right)$ representa o tempo que o veículo leva para ir do centro do vermelho do semáforo $S_{j_{k}}$ até o centro do vermelho do semáforo $S_{j_{m}}$ e $p$ é o número de trechos entre os semáforos das interseções do loop.

$c_{l}\left(j_{1}, \ldots, j_{p}\right)$ é uma variável inteira medida em ciclos, representando o tempo que o veículo levaria para percorrer o trecho do centro do vermelho do semáforo $S_{j_{1}}$ e voltar para o mesmo algum centro de vermelho posterior. Considerando o percurso no sentido contrário, tem-se uma expressão análoga: 
$c_{l}\left(j_{1}, \ldots, j_{p}\right)=\bar{\phi}\left(j_{1}, j_{2}\right)+\ldots+\bar{\phi}\left(j_{p}, j_{1}\right)+1 / 2 p$

Adicionando as expressões (9) e (10), e sabendo que

$m\left(j_{k}, j_{m}\right)=\phi\left(j_{k}, j_{m}\right)+\bar{\phi}\left(j_{k}, j_{m}\right)$, tem-se:

$2 c_{l}\left(j_{1}, \ldots, j_{p}\right)=m\left(j_{1}, j_{2}\right)+\ldots+m\left(j_{p}, j_{1}\right)+p=$ número inteiro

Como $p$ é inteiro, a restrição simplificada fica:

$2 c_{l}\left(j_{1}, \ldots, j_{p}\right)=m\left(j_{1}, j_{2}\right)+\ldots+m\left(j_{p}, j_{1}\right), \forall l=1,2, \ldots, L$

(7) Restrições de tempo de vermelho para alguns semáforos;

Às vezes, interessa controlar o tempo de vermelho de alguns semáforos importantes. Neste caso, consideram-se limites para estes tempos da forma:

$R M I M_{i j} \leq r_{i j} \leq R M A X_{i j}, \quad$ para alguns i's e s's, no sentido

$$
\text { outbound; }
$$

$R M I M_{i j} \leq \overline{r_{i j}} \leq R M A X_{i j} \quad$ para alguns i's e s's, no sentido

inbound;

(8) Restrições de positividade

Todas as variáveis de decisão são positivas, algumas podem assumir valores reais e outras só inteiras, a saber:

Variáveis Reais:

$b_{i} \geq 0, z \geq 0, u_{i} \geq 0, \bar{u}_{i} \geq 0, w_{i j} \geq 0, \bar{w}_{i j} \geq 0, \quad i=0, \ldots, q \quad j=1, \ldots J_{i}$

Variáveis Inteiras:

$m\left(j_{k}, j_{m}\right) \geq 0, \quad c_{l}\left(j_{1}, \ldots, j_{p}\right) \geq 0$ 


\subsection{Sincronização dos Semáforos}

Executando o modelo, obtém-se a sincronização através da diferença de fase entre semáforos, que pode ser calculada da forma:

Seja $\theta_{i}\left(j_{1}, j_{2}\right)$ a fase relativa dos semáforos $S_{j_{i}}$ e $S_{j_{2}}$ da artéria $i$ medida pelo tempo do centro do vermelho de $S_{j_{i}}$ para o próximo centro de vermelho de $S_{j_{2}}$.

$$
\theta_{i}\left(j_{1}, j_{2}\right)=\text { mantissa } \phi\left(j_{1}, j_{2}\right)
$$

Fazendo este cálculo relativo ao primeiro semáforo da artéria $i$, tem-se:

$\theta_{i}(1, j)=$ mantissa $\left[w_{i 1}-w_{i j}+\sum_{k=1}^{j-1} t\left(j_{k}, j_{k+1}\right)+\frac{1}{2}\left(r_{i 1}-r_{i j}\right)\right] \quad, \quad j=2, \ldots, J_{i}$

O conjunto $\left\{\theta_{i}(1, j), j=2, \ldots, J_{i}\right\}$ é chamado de sincronização dos semáforos da artéria $i$. A partir destes valores, a sincronização pode ser executada.

\subsection{Dimensão e execução do modelo}

Em relação ao número de variáveis do modelo, tem-se:

$$
\begin{aligned}
& b^{0}, \ldots, b^{q} \\
& z \\
& u_{0}, u_{1}, \ldots, u_{q} \\
& \bar{u}_{0}, \bar{u}_{1}, \ldots, \bar{u}_{q} \\
& w_{01}, w_{11}, \ldots, w_{1 j_{1}}, w_{q 1}, \ldots, w_{q j_{q}} \\
& \bar{w}_{01}, \bar{w}_{11}, \ldots, \bar{w}_{1 j_{1}}, \bar{w}_{q 1}, \ldots, \bar{w}_{q j_{q}} \\
& m_{1}(1,2), \ldots ., m_{1}\left(J_{1}-1, J_{1}\right), \ldots ., m_{q}(1,2), \ldots ., m_{q}\left(J_{q}-1, J_{q}\right) \\
& c_{1}, \ldots, c_{L} \\
& r_{11}, \ldots . . r_{1 J 1}, \ldots \ldots ., r_{q 1}, \ldots \ldots ., r_{q J_{q}}
\end{aligned}
$$

O número de variáveis está representado na Tabela 1.

Tabela 1: Número de variáveis do modelo 


\begin{tabular}{c|c}
\hline VARÍAVEL & NÚMERO DE VARIÁVEIS \\
\hline $\mathrm{b}:$ & $\mathrm{q}+1$ \\
\hline $\mathrm{z}:$ & $2 \mathrm{q}$ \\
\hline $\mathrm{u}:$ & $2 q\left(J_{1}+\ldots+J_{q}\right)=2 q\left(J_{1}+\ldots+J_{q}\right)$ \\
\hline $\mathrm{w}:$ & ${ }^{2}-1+\ldots+J_{q}-1=\sum_{i=1}^{q} J_{1}-q$ \\
\hline $\mathrm{m}:$ & $\mathrm{L}$ \\
\hline $\mathrm{c}:$ & $\sum_{i=1}^{q} J_{i}$ \\
\hline $\mathrm{r}:$ & ${ }^{2}$
\end{tabular}

O número total será então $2 q+4+L+3 J+J J$, onde:

$q:$ número de artérias principais

$L$ : número de restrições - loops

$\sum J_{i}=J$ : número de todos os semáforos da rede

$J J$ : número de semáforos com controle de tempo de vermelho

Em relação ao tamanho da matriz de restrições, suas dimensões serão da ordem representada na Tabela 2 . Neste caso, tem-se um total de $4 q+6+3 J+L+J J$ restrições.

Para avaliar o tamanho do problema, considerando uma rede com 50 artérias a serem sincronizadas e 10 semáforos em cada uma, formando 20 loops na malha e $J J=20$. Neste caso, 0 número de variáveis será de 1644 variáveis, sendo que 470 variáveis são inteiras. O número de restrições é de 1746.

Em função do número possível de variáveis e restrições, que podem gerar um problema de médio porte, a sua execução vai exigir computadores pessoais de alta performance.

Tabela 2: Número de restrições do modelo

\begin{tabular}{c|c}
\hline RESTRIÇÃO & NÚMERO DE RESTRIÇÕES \\
\hline (R1) & q restrições
\end{tabular}




\begin{tabular}{c|c} 
(R2) & 2 restrições \\
\hline (R3) & $4(q+1)$ restrições \\
\hline (R4) & $q\left(\sum_{i=1}^{q} J_{i}\right)$ restrições \\
\hline (R5) & $\sum_{i=1}^{q} J_{i}-q$ restrições \\
\hline (R6) & L restrições \\
\hline (R7) & JJ, sendo JJ<<J restrições \\
\hline
\end{tabular}

Vários algoritmos podem ser utilizados para resolver este modelo de programação linear mista. Sugere-se o Método Branch and Bound ou alguma variante associada a uma heurística. Tais métodos são bastante usados em problemas deste porte e tipo.

A forma geral da matriz de restrições pode ser observada na Tabela 3.

Tabela 3 : Matriz de restrições do modelo.

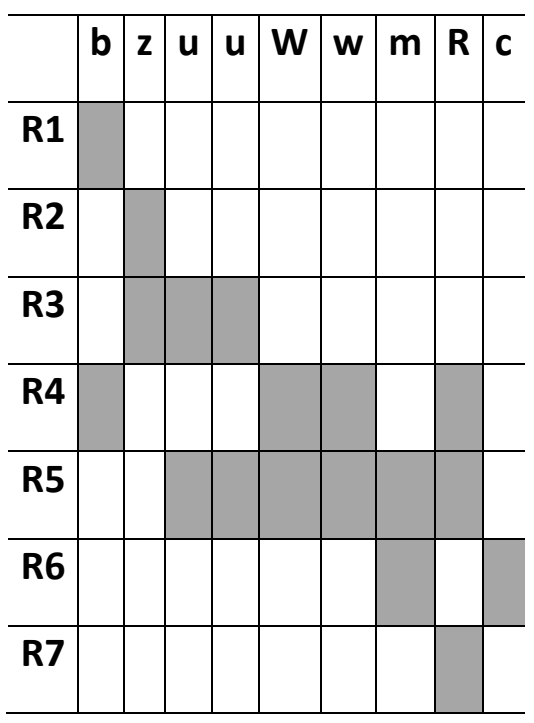

\section{CONSIDERAÇÕES FINAIS}


Espera-se que a implementação do modelo matemático numa área no trânsito de Curitiba ocorra uma melhora na estrutura da malha viária e uma maior organização no tráfego, refletindo em aumento da segurança no trânsito. Com os resultados obtidos, espera-se que o fluxo no trânsito melhore, promovendo a diminuição do stress do motorista, e assim, em um ambiente mais agradável, o número de acidentes tenderá a diminuir consideravelmente. A redução de acidentes consiste em benefício indireto da sincronização dos semáforos, quando associado a medidas de controle de velocidade, de adequação da capacidade das vias e de respeito ao pedestre e ciclistas.

Algumas cidades obtiveram ótimos resultados com a implantação de sincronização semafórica, como Recife, onde foram sincronizados 21 semáforos na Avenida Boa Vista, havendo um ganho de $22 \%$ a $50 \%$ de velocidade. Houve também ganhos no tempo de percurso, como na Avenida Bernardo Vieira de Melo, onde o trajeto, antes feito em 17min30s, foi reduzido para 6 min53s. Outro exemplo é a cidade de Santos, onde o congestionamento foi reduzido em até $40 \%$ em algumas avenidas (JORNAL DO COMÉRCIO, 2001).

O crescimento acelerado de Curitiba tem tornado o trânsito da cidade desorganizado e lento. Sabendo dos resultados positivos da sincronização em outras cidades nota-se a necessidade da implantação do sistema em Curitiba.

O passo seguinte seria partir para a automação dos resultados através de um sistema de controle automático à distância, conforme já proposto em outros trabalhos, como o citado por Rosseti (1998).

\section{REFERÊNCIAS BIBLIOGRÁFICAS}

1. BRASIL. Instituto Brasileiro de Geografia e Estatística - IBGE. Produção Agrícola Municipal.2010. Disponível em: http://www.sidra.ibge.gov.br/. Acesso em : 10 mai. 2011.

2. CNT-Código Nacional de Trânsito. LEI № 9.503, DE 23 DE SETEMBRO DE 1997.

3. COHEN, S. L., LIU C. C. The bandwidth-constrained TRANSYT signal-optimization program. Transportation Research Record, v. 1057, p. 1-7, 1986.

4. COHEN, S. L., PILLAI R. S. A restricted Branch and Bound approach for generating maximum bandwidth signal timing plans for traffic networks. Transportation Research, v. 32, n. 8, p. 517-529, 1998.

5. GARCIA, H. C. e GARAVELlO, T. M. Geografia do Brasil, Dinâmica e Contrastes, Editora Scipione, 6a edição, São Paulo, 1995.

6. GARTNER, N. H. Constraining relations among offsets in synchronized signal networks. Transportation Science, v. 6, p. 88-93, 1972.

7. GARTNER, N. H., LITTLE, J. C. and GABBAY, H. Optimization of traffic signal settings by mixedinteger linear programming. Part I: the network coordination problem. Transportation Science, v. 9, p. 321-343, 1975 a.

8. JORNAL DO COMÉRCIO. Recife 12 de dezembro de 2001. 
9. LITTLE, J. C. The synchronization of traffic signals by mixed-integer linear programming. Operations Research, v. 14, p. 568-594, 1966.

10. LITTLE, J. C., KELSON M. D. and GARTNER, N. H. Maxband: A program for setting signals on arteries and triangular networks. Transportation Research Record ,v.795, p. 40-46, 1981.

11. MORGAN, J. T. e LITTLE, J. D. C. Synchronization traffic Signals for Maximal Bandwith, Operations Research, v. 12, p. 896-912, 1964.

12. PNT: Política Nacional de Trânsito - Versão para debate apresentado no $12 \circ$ Congresso Brasileiro de Transportes e Trânsito, ANTP, 14 a 18 de Junho de 2001, Recife.

13. PORTO Jr, W., FERREIRA, A. C. M. Avaliação e sugestões para um plano semafórico. Anais do VII Congresso da ANPET, São Paulo, v. 2, 831-840, 1993.

14. ROSSETI, R.J.F. Um ambiente para suporte à simulação de sistemas de tráfego urbano, dissertação de mestrado, CPGCC, UFRGS, 1998.

15. WILSON, R. J. e WATKINS, J. J. Graphs an introductory approach. New York: Willey, 1990. 